\title{
Author Index Vol. 14, 1994
}

Abbal,M. 187 Adams, J.E. 19 Ahmad, G.S. 6 Ahmed, T.M. 295 Aliotta, G. 377, 399 AlKhayat,R. 113 Almirall,J. 192 Amici, G. 216 Angeletti, L.R. 282 Antonello,A. 432 Appel,R.G. 90,230 Arakawa, M. 236 Arduino, MJ. 85 Arranz Arija, J.A. 148 Ashizawa, A. 142 Ataiipour, Y. 213 Avagliano, F. 494

Balk,A.H. 1 Ballardie, F.W. 233 Bang,B.K. 72,220 Barratt, L.J. 47 Basso, G. 157 Bastiani, T. de 302,317 Beck-Sague, CM. 85 Bellinghieri, G. 76 Bencini,P.L. 121 Bernabeo, R.A. 313 Bianchetti, M.G. 201 Bland, L,A. 85 Bocci,C. 216 Bonomini, V. 361 Boogaerts, M. 197 Borsatti,A. 432 Bos,E. 1 Brillet,G. 81 Buchner, L.M. 226 Buckalew, V.M., Jr. 90 Buller,G.K. 223 Bunker, D. 81 Burkart, J.M. 90

Cameron, J.S. 365,452 Campese, V.M. 55 Campieri, C. 361 Capasso,G. 377,426 Caputo,R. 121 Card,M.L. 9 Carrion, J.R. 148 Cavarra,B. 282 Chan,E.L.P. 295 Chan, J.C.M. 295 Chang, Y.S. 72,220

Cheng, A.B. 182 Chevalier, R.L. 461 Chicca,S. 106 Chieffi,G. 458 Chiozza, M.L. 157 Cirillo,M. 426 Coen,G. 106 Coleman, M. 47 Collins, J.J. 169 Colussi, G. 127 Combarnous, F. 213 Conte,G. 257 Costerton, J.W. 37 Courdavault, L. 113 Coward, A. 488

Dasgupta, A. 162 David, L. 213 DeAntoni,E. 106 De Ferrari, M.E. 127 De Matteis Tortora, M. 412, 418 Deray,G. 81 De Santo, N.G. 255, 377, 426, 448, 488, 494 Diamandopoulos, A.A. 239 Dianzani, M.U. 330 DiSanza,P. 106 D’Onorio,B. 477 Dorpel, M.A.v.d. 1 Durand,D. 187

Edwards, B.D. 233 Eknoyan,G. 255,270,488,494 El-Shahawy, MA. 55 English, J.A. 371

Federspil,G. 337 Feld,L.G. 169 Fellner, S.K. 64 Fine,L.G. 371 Fogazzi, G.B. 452 Fragale, M.R. 14 Freedman, B.I. 90 Freeman, M.R. 173 Freemont, A.J. 19 Frith, P.A. 47

Gadallah, M.F. 55 Gallo,L. 494 Gameren, I.I. v. 67 Garcia, F.R. 148 Gejyo, F. 236

Gemelli,M. 76 Glowacki, L.S. 9 Gokal,R. 19 Goldstein, M.B. 173 Gottschalk, C.W. 320 Grandesso, S. 216

Hammerberg, O. 9 Haramoto, T. 30 Harlos,J. 436 Heidland,A. 436 Hellemans,L. 197 Hierholzer, C. 467 Hierholzer, J. 467 Hierholzer, K. 344,467 Hodsman, A.B. 9 Hutchison, AJ. 19

Ikeda,S. 30 Indudhara, R. 154 Inenaga, T. 142 Ise,M. 207 Ishida, K. 236 Ishimitsu, T. 60 Iskandar, S.S. 90

Jackson, B.M. 85 Jacobs, C. 81 Jacquiaud, C. 81 Jallous,H. 302,317 Jankova, T. 246 Jarvis,W.R. 85 Jong, P.E. de 67

Kanaka, C. 201 Kawano,Y. 142 Kedes,L. 41 Khauli,R,B. 154 Khoury,A.E. 37 Kim,B.K. 220 Kim, Y.S. 72,220 Kimura,G. 142 Kinne,R.K,H. 355 Kinne-Saffran, E. 355 Koide,H. 136 Kopple,J.D. 279 Kornberg, A.E. 169 Küster,S. 443

Lai,F.M. 99 Lai, K.-N. 99,182 Lamendola, M.G. 448 Lampa,E. 384

Langer, A. 173 Langford, L.R. 47 Laville,M. 213 Leung, C.-B. 99,182 Leung, J.C.K. 99 Li,P.K.T. 99,182 Llibre,J. 192 Loffreda,A. 384 Lucas, M.F. 95 Lui,S.-F. 99,182

Macaluso, M. 127 McGhie,K. 9 McHale,J. 233 McNeill, V. 9 Makino,H. 30 Malik, S. 162

Mangrella, M. 384 Manni, M. 106 Marandola,P. 302,317 Marcen, R. 95 Maresca, M.C. 216 
Marketos, S.G. 239,264 Massry,S.G. 41,255,307 Matsuoka, H. 142 Mattoo,T.K. 6 Mawer,E.B. 19 Mazzaferro, S. 106 Meeter, K. 1 Meillet,D. 81 Melillo,L. 423 Menarguez, F.J. 148 Menon, M. 154 Meraw, J. 9 Meyer, L. 85 Michel, C. 113 Mignon, F. 113 Mignot,L. 81 Minetti,L. 127 Miyazaki,T. 207 Modesto, A. 187 Monardo, P. 76 Montagnino, G. 121 Montini,G. 157 Mottola,A. 216 Mullis, P.E. 201 Murer, L. 157 Musitelli,S. 302,317 Musolino, R. 76 Mydlow,P.K. 169

Nakamura, Y. 60 Navarro, J.F. 95

499

Nenov,D. 246 Newstead, C. 233 Niwa,T. 207 Nolph,K,D. 145

Ohkubo,M. 60 Ohmuro,H. 136 Oldoni,M. 483 Omae,T. 142 Ongkingco, J.R.C. 14 Onorato, L. 106 Ortuño,J. 95 Ota,Z. 30

Palmero,J.R. 290 Papatheofanis, J.A. 64 Pasca,M. 478 Paskalev,D. 246 Pasquali, M. 106 Peheim,E. 201 Perazella, M.A. 223 Petrucelli, R.J., II 259 Pérez-Manga, G. 148 Perna,A. 494 Pollio,A. 377,399 Ponticelli, C. 121,452 Potters, H. 9 Preston, C.A.K. 37

Raymond, F. 81 Reid,G. 37 Rensma, P.L. 67 Rippa-Bonati, M. 432 Ritz,E. 443,452 Rivera, M. 95 Roffi,M. 201 Rombolà,G. 127 Rossi, F. 384 Rostaing,L. 187 Roux,P. 113 Ruley,E.J. 14

Sabatini,S. 391 Sanai,T. 142 Sardella,D. 106 Savica, V. 76 Scarpa, A. 157 Schena,F.P. 325 Scolari, M.P. 361 Shah,D.J. 226 Sherin,K. 169 Sicolo,N. 337 Singh, N. 173 Smogorzewski, M. 41

Snauwaert, J. 197 Speroni,A. 302,317 Spurio,A. 106

Steiner,M. 246 Stella, N.C. 76 Stirling, J.W. 47 Stitt,L.W. 9 Strumia,S. 377

Tarantino, A. 121 Tardio,J. 148 Tchankova, P. 246 Tejedor, A. 148 Teodori, T. 216 Teruel,J.L. 95 Tian,J. 41 Timio,M. 473 Tizianello, A. 383 Tomino, Y. 136 Triolo,O. 76 Trivedi,H.S. 145

Trombetta,M. 106 Turner, M.E. 14

Veciana, L. 192 Veiga,P. 169 Villafruela, J.J. 95 Virga,G. 216 Viron,B. 113 Voyiatzakis, E. 239

Wang, A. 99 Wang, M. 295 Weimar, W. 1 Whitehouse, R.W. 19 Wieslander, J. 30 Williams, N.P. 226 Williams, W. 226 Wolf,J.de 67 Wong,K.-C. 182

Yagi,H. 60 Yagi,S. 60 Yang,C.W. 72,220 Yang,K,H. 72,220 Yoon, Y.S. 72,220 Yu,B. 64

Zacchello, F. 157 Zacchello, G. 157 Zachée,P. 197 Zech,P. 213 Zeier,M. 443 Zietse, R. 1 Zijlstra, J.G. 67 Zuccoli, M. 361

500

Author Index 